\title{
The Roles of Procalcitonin, C-Reactive Protein and Erythrocyte Sedimentation Rate in Predicting Bacteremia
}

\author{
Faqi $\mathrm{MK}^{*}$, Al-Qahtani $\mathrm{M}^{2,3}$ and Elmusharaf $\mathrm{K}^{4}$ \\ ${ }^{1}$ Orthopedic Resident, Department of Orthopedics, Royal Medical Services, Bahrain Defence Force Hospital, Riffa, \\ Bahrain \\ ${ }^{2}$ Clinic Microbiologist, Head of Microbiology, Pathology and Laboratory Medicine Department, Royal Medical \\ Services, Bahrain Defence Force Hospital, Riffa, Bahrain \\ ${ }^{3}$ Senior Microbiology Lecturer, Royal College of Surgeons in Ireland- Medical University of Bahrain, Behrain \\ ${ }^{4}$ Lecturer in Epidemiology \& Public Health Medicine, Royal college of Surgeons in Ireland, RCSI Bahrain
}

*Corresponding author: Faqi MK, Orthopedic Resident, Department of Orthopedics, Royal Medical Services, Bahrain Defence Force Hospital, Riffa, Bahrain, E-mail: drmfaqi@gmail.com

Citation: Faqi MK, Al-Qahtani M, Elmusharaf K (2015) The Roles of Procalcitonin, C-Reactive Protein and Erythrocyte Sedimentation Rate in Predicting Bacteremia. J Immunol Infect Dis 2(2): 205. doi: 10.15744/2394-6512.1.206

Received Date: August 10, 2015 Accepted Date: October 28, 2015 Published Date: October 30, 2015

\begin{abstract}
Background and objectives: The early differentiation between infectious and non-infectious sepsis remains a challenge due to the lack of a reliable, ready available and quick biomarker of bacterial sepsis. This study aims to determine the diagnostic accuracies of procalcitonin (PCT), C-reactive protein (CRP) and erythrocyte sedimentation rate (ESR) as individual and combined predictors of bacterial sepsis, when compared to the gold standard microbiological cultures.

Patients and methods: Forty-three patients were included in this study, provided they fulfilled the criteria established by ACCP/SCCM consensus conference for systemic inflammatory response syndrome (SIRS) and sepsis. The primary outcome was to determine the best predictor of bacteremia. The diagnostic accuracies of PCT, CRP and ESR, individually and in combinations were calculated.

Results: Among 43 patients, 27 yielded positive blood cultures. Procalcitonin, CRP and ESR, individually, were able to predict $74.1 \%$ ( $95 \%$ CI; 0.553 to 0.868 ), $66.7 \%$ (95\% CI; 0.478 to 0.814 ) and $70.4 \%$ (95\% CI; 0.515 to 0.842 ), of positive blood cultures, respectively. In comparison, interpreting PCT \& CRP, PCT \& ESR and PCT, CRP \& ESR in combinations were able to predict $51.9 \%$ (95\% CI; 0.340 to 0.693), $59.3 \%$ ( $95 \%$ CI; 0.407 to 0.755 ), $37.0 \%$ (95\% CI; 0.215 to 0.558 ).

Conclusion: Procalcitonin, on its own, is the best predictor of bacteremia. Although PCT is an indispensible biomarker for the early prediction of bacteremia, careful evaluation of patients' clinical status and other laboratory markers are also crucial.

Keywords: Bacteremia; C-reactive protein; Erythrocyte Sedimentation Rate; Procalcitonin; Sepsis

List of Abbreviations: AUC: Area Under Curve; CRP: C-Reactive Protein; ESR: Erythrocyte Sedimentation Rate; PCT: Procalcitonin; ROC: Receiver Operating Characteristic; SIRS: Systemic Inflammatory Response Syndrome
\end{abstract}

\section{Introduction}

Despite the growing advancements of infection control and antibiotic therapy; the incidence of sepsis in developed countries has been persistently high reaching up to $30 \%$ of all admissions to intensive care units with a mortality rate of $62 \%$, as reported in the SOAP study [1]. Such unfavorable prognosis for septic patients appears to be multi-factorial; with delayed diagnosis and subsequent treatment being the most significant factor. One obstacle is the accurate differentiation between infectious sepsis and non-infectious causes of sepsis i.e. systemic inflammatory response syndrome (SIRS). Another obstacle is the lack of readily available, quick and highly sensitive and specific parameter for diagnosing infectious sepsis [2-4].

The diagnostic criterion for sepsis according to ACCP/SCCM Consensus Conference in 1992 was broadly classified under the term SIRS which constitutes symptoms of pyrexia, tachycardia, tachypnea and leucocytosis [5]. However, SIRS could result from both infectious and non-infectious etiologies of systemic inflammation, which necessitates accurate differentiation and prompt management accordingly. The current practice uses microbiological cultures for differentiating the underlying bacterial from non-bacterial causes. Although microbiological culture is the gold standard test, it is time-consuming and uneconomical causing delayed treatments, subsequently leading to poor prognosis along with higher costs on healthcare systems. 
Survival rates of septic patients vary widely, requiring immediate intervention despite the delayed microbiological culture results. The empirical use of antibiotics is effective if the sepsis is of bacterial etiology. However, for non-bacterial sepsis, such practice would increase antibiotic resistance, morbidity and eventual mortality. Therefore, identifying a bacterial/non-bacterial etiology of sepsis is crucial. As a result, recent studies have investigated biomarkers such as procalcitonin (PCT) [4,6-8] and C-reactive protein (CRP) $[9,10]$ for the early detection of bacteraemia in septic patients.

Procalcitonin is a 116-amino acid prohormone of calcitonin. It is produced by parafollicular C cells of the thyroid gland and neuroendocrine cells in the lung and intestine [11]. The level of procalcitonin in healthy individuals is usually undetectable [12]. High PCT concentrations peaks at the onset of inflammation indicating severe bacterial sepsis or septic shock, thus allowing differentiation between infectious causes of sepsis from non-infectious ones. Half-life of PCT is 25-30 hours in serum, which allows for the proper assessment of response to therapy [11].

Other common laboratory biomarkers that were widely studied are CRP and erythrocyte sedimentation rate (ESR). When considering the reported sensitivity and specificity of PCT, CRP and ESR, a large discrepancy exists. Studies of PCT reported sensitivity and specificity ranges of 74.8-100 and 70-100\%, respectively [2]. On the other hand, whilst the wide use of CRP and ESR as adjunctive serum biomarkers of inflammation and sepsis, many studies have questioned their roles, particularly due to an inconsistency in their reported diagnostic accuracy parameters [13-15].

This prospective cohort study aims to determine the diagnostic accuracy of PCT, CRP and ESR as individual and combined indicators of bacterial sepsis, when compared to the gold standard microbiological cultures.

\section{Materials and Methods}

\section{Patients' selection}

The following prospective study was approved by the research and ethics committee of Bahrain Defence Force (BDF) military hospital. The study was conducted in the medical ward and Intensive Care Unit (including Cardiac Care Unit) of the BDF hospital in Bahrain between April and May 2015. Patients, of both genders, suffering SIRS, sepsis, severe sepsis or septic shock according to the criteria established by ACCP/SCCM Consensus Conference, were included in this study [5]. Procalcitonin, CRP, ESR and microbiological cultures were obtained for each blood sample within the same time ( \pm 24 hours). Patients on antibiotics at time of blood collection or immunosuppressive drugs; or patients with major trauma, severe burns or recent surgery were excluded. Patients diagnosed with small cell lung cancer or thyroid carcinoma (C-cell) were also excluded.

\section{Procalcitonin measurement and interpretation}

To prepare the blood samples for PCT measurement, EDTA-plasma was separated from whole blood by centrifugation. PCT was measured semi-quantitatively using the "PCT-Q" (BRAHMS Diagnostica, Berlin, Germany). Six drops of plasma were dropped into the round cavity of the assay and left for 30 minute of incubation at room temperature. Once the incubation period is complete, the validity of the test is determined by checking that the control band is visible. To determine the PCT concentration, the color intensity of the test band is compared to the color blocks of the reference card supplied with the kit. The color intensity of the test band corresponds to the PCT concentration as four categories provided by the reference scale. The interpretations and risk of progression to severe sepsis for each category is shown in Table 1.

\begin{tabular}{|c|c|c|}
\hline PCT & Interpretation & $\begin{array}{c}\text { Risk of progression to severe systemic } \\
\text { infection (severe sepsis) }\end{array}$ \\
\hline$<\mathbf{0 . 5} \boldsymbol{\mu g} / \mathrm{L}$ & $\begin{array}{c}\text { Local bacterial infection is possible Systemic infection (sepsis) is not } \\
\text { likely }\end{array}$ & Low \\
\hline $\mathbf{0 . 5}-\mathbf{2} \boldsymbol{\mu g} / \mathbf{L}$ & $\begin{array}{c}\text { Systemic infection (sepsis) is possible, but various conditions are } \\
\text { known to induce PCT as well }\end{array}$ & Moderate \\
\hline $\mathbf{2 - 1 0} \boldsymbol{\mu g} / \mathbf{L}$ & $\begin{array}{c}\text { Systemic infection (sepsis) is likely High risk for progression to } \\
\text { severe systemic infection (severe sepsis) }\end{array}$ \\
\hline$>\mathbf{1 0} \boldsymbol{\mu g} / \mathbf{L}$ & $\begin{array}{c}\text { Important systemic inflammatory response, almost exclusively due } \\
\text { to severe bacterial sepsis or septic shock }\end{array}$ & $\begin{array}{c}\text { High likelihood of severe sepsis or septic } \\
\text { shock }\end{array}$ \\
\hline
\end{tabular}

Table 1: Interpretation of PCT-Q (BRAHMS) values and risk of progression to severe sepsis, adapted from Meisner et al. [7]

\section{CRP, ESR and blood cultures}

Serum concentrations of CRP were quantified by latex-enhanced nephelometry using BNII nephelometer system (Dade Behring, Milan, Italy; now Siemens Inc, Germany). CRP levels above $0.01 \mathrm{~g} / \mathrm{L}$ were considered abnormal. ESR was measured using the Westergren method using the Sediplast ESR system (Polymedco Inc, Cortlandt Manor, NY, USA). ESR above 15 mm/hour was considered abnormal. The BACTEC 9240 automated blood culture system (Becton Dickinson Diagnostics, Sparks, MD, USA) was used to process blood cultures. Coagulase-negative staphylococcus grown on blood culture samples were considered as contaminants; and therefore rendered as negative blood cultures. 


\section{Statistical analysis}

Statistical data analyses were performed using SPSS-19 software. The diagnostic accuracies of PCT, CRP and ESR, individually and in combinations, were assessed by calculating the sensitivity, specificity, positive and negative likelihood ratios, diagnostic odds ratio, and positive and negative predictive values (Table 6). Statistical significance was considered at $\mathrm{P}<0.05$ for all parameters. Confidence intervals of $95 \%$ were expressed for uncertainty. A ROC curve was drawn for the diagnostic tests individually and in combinations (Figure 1). Area under the curves (AUC) for positive microbiological blood cultures of PCT, CRP and ESR, individually and in combinations were calculated (Table 7).

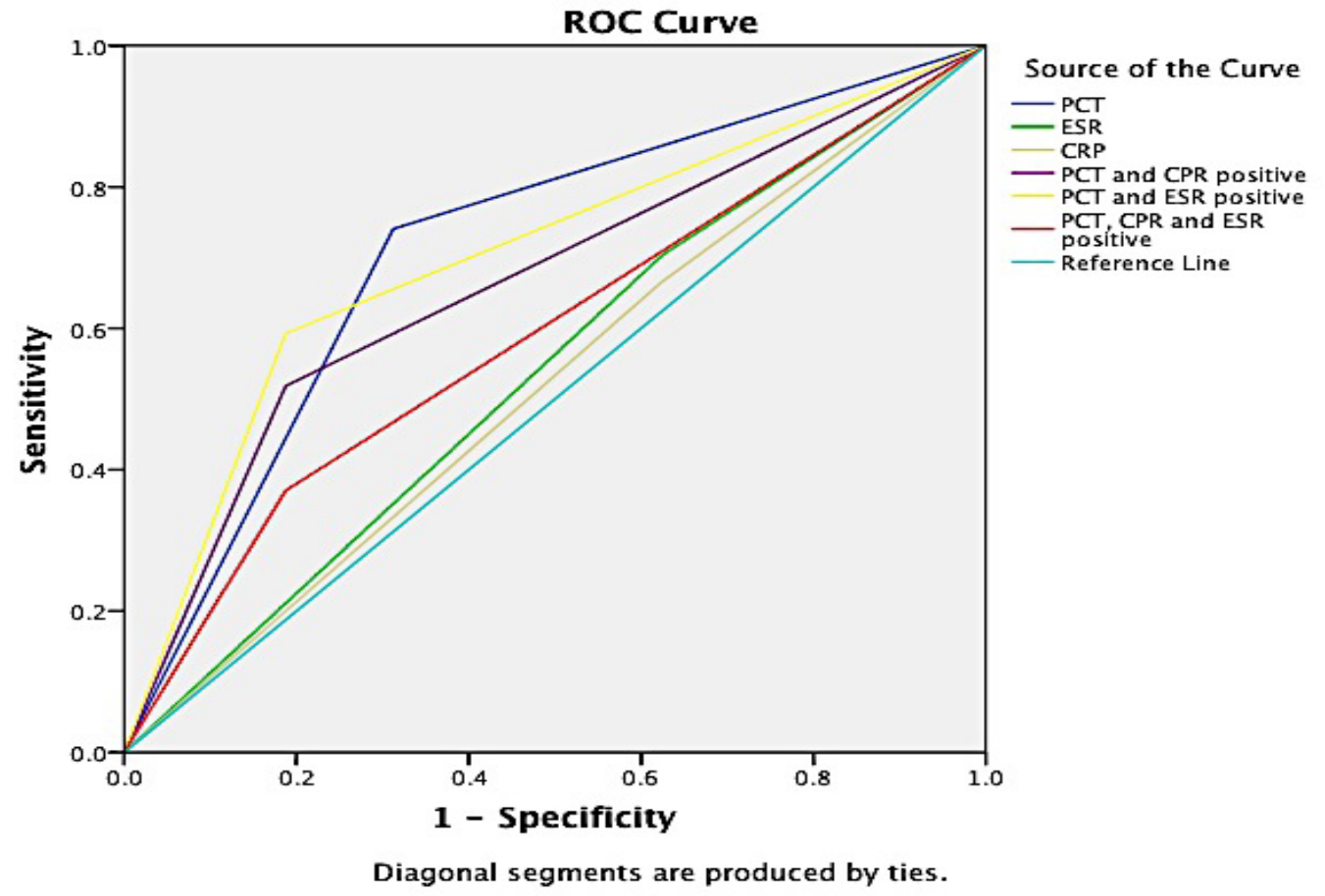

Figure 1: Receiver operating characteristics (ROC) for positive microbiological blood cultures of PCT, CRP and ESR, individually and in combinations

\section{Results}

A total of 45 blood samples were collected from 45 patients. Two patients were excluded from this study because of the presence of contaminants in the blood culture samples. The mean, median and range of age of the population studied were 57, 61 and 25-82 years, respectively. Among 43 patients, 27 (62.79\%) had confirmed bacteremia (confirmed by blood cultures). Of the 27 patients with proven bacteremia, Gram-positive bacteria was detected in 16 (59.3\%) samples, whilst Gram-negative bacteria was detected in 11 samples (40.7\%) (Table 2). PCT, CRP and ESR were able to correctly predict positive blood cultures in 20, 18 and 19 samples, respectively (Table 3). Combinations of PCT and CRP; PCT and ESR; and PCT, CRP and ESR were able to correctly predict positive blood cultures in 14 (51.9\%), 16 (59.3\%) and 10 (37\%) samples, respectively. Patient's characteristics are presented in Table 4. Differences in characteristics between positive and negative microbiological blood cultures are shown in Table 5.

\begin{tabular}{|c|c|}
\hline Bacteria & Number (\%) \\
\hline Gram-positive & $16(59.3 \%)$ \\
\hline Staphylococcus aureus (S. aureus) & 8 \\
\hline Streptococcus pyogenes (S. pyogenes) & 2 \\
\hline Streptococcus pneumoniae & 6 \\
\hline Gram-negative & $11(40.7 \%)$ \\
\hline Klebsiella spp & 3 \\
\hline Escherichia coli (E. coli) & 7 \\
\hline Pseudomonas aeruginosa & 1 \\
\hline
\end{tabular}

Table 2: Gram-positive Vs. Gram-negative bacteria isolated from blood cultures

The diagnostic accuracies of PCT, CRP and ESR, individually and in combinations, for positive blood cultures are shown in Table 6. ROC curves of PCT, CRP and ESR, individually and in combinations in differentiating positive blood cultures are shown in Figure 1. PCT, individually, showed the highest AUC of 0.714 . The AUC for positive microbiological blood cultures of PCT, CRP and ESR, individually and in combinations are presented in Table 7. 


\begin{tabular}{|c|c|c|c|}
\hline & $\begin{array}{l}\text { Positive Blood } \\
\text { Cultures }\end{array}$ & $\begin{array}{l}\text { Negative Blood } \\
\text { Cultures }\end{array}$ & Total \\
\hline PCT $>2$ & 20 & 5 & 25 \\
\hline $\mathrm{PCT}<2$ & 7 & 11 & 18 \\
\hline \multirow[t]{2}{*}{ Total } & 27 & 16 & 43 \\
\hline & $\begin{array}{l}\text { Positive Blood } \\
\text { Cultures }\end{array}$ & $\begin{array}{l}\text { Negative Blood } \\
\text { Cultures }\end{array}$ & Total \\
\hline $\mathrm{CRP}>0.01 \mathrm{~g} / \mathrm{L}$ & 18 & 10 & 28 \\
\hline $\mathrm{CRP}<0.01 \mathrm{~g} / \mathrm{L}$ & 9 & 6 & 15 \\
\hline \multirow[t]{2}{*}{ Total } & 27 & 16 & 43 \\
\hline & $\begin{array}{l}\text { Positive Blood } \\
\text { Cultures }\end{array}$ & $\begin{array}{c}\text { Negative Blood } \\
\text { Cultures }\end{array}$ & Total \\
\hline $\mathrm{ESR}>15 \mathrm{~mm} / \mathrm{hr}$ & 19 & 10 & 29 \\
\hline $\mathrm{ESR}<15 \mathrm{~mm} / \mathrm{hr}$ & 8 & 6 & 14 \\
\hline Total & 27 & 16 & 43 \\
\hline
\end{tabular}

Table 3: Comparison between micriobiological blood cultures and procalcitonin, CRP and ESR in diagnosing bacterial sepsis

\begin{tabular}{|c|c|c|c|}
\hline \multicolumn{2}{|c|}{ Patients' characteristics } & Frequency & Percent \\
\hline \multirow{3}{*}{ Age groups } & $<60$ years & 18 & 41.9 \\
\cline { 2 - 4 } & 60 and above & 25 & 58.1 \\
\hline \multirow{3}{*}{ Gender } & Male & 24 & 55.8 \\
\cline { 2 - 4 } & Female & 19 & 44.2 \\
\hline \multirow{2}{*}{ PCT } & Positive & 25 & 58.1 \\
\cline { 2 - 4 } & Negative & 18 & 41.9 \\
\hline \multirow{2}{*}{ ESR } & Positive & 29 & 67.4 \\
\cline { 2 - 4 } & Negative & 14 & 32.6 \\
\hline \multirow{2}{*}{ CRP } & Positive & 28 & 65.1 \\
\cline { 2 - 4 } & Negative & 15 & 34.9 \\
\hline \multirow{2}{*}{ Culture } & Positive & 27 & 62.8 \\
\cline { 2 - 4 } & Negative & 16 & 37.2 \\
\hline
\end{tabular}

Table 4: Patients characteristics

\begin{tabular}{|c|c|c|c|c|c|c|c|}
\hline \multirow{2}{*}{\multicolumn{2}{|c|}{ Patients' characteristics }} & \multicolumn{2}{|c|}{ Positive Culture $(\mathrm{n}=27)$} & \multicolumn{2}{|c|}{ Negative Culture $(n=16)$} & \multirow{2}{*}{ Total } & \multirow{2}{*}{$P$ value } \\
\hline & & Frequency & Percent & Frequency & Percent & & \\
\hline \multirow{2}{*}{ Age groups } & $<60$ years & 10 & $55.6 \%$ & 8 & $44.4 \%$ & 18 & \multirow{2}{*}{0.41} \\
\hline & $60+$ & 17 & $68 \%$ & 8 & $32 \%$ & 25 & \\
\hline \multirow{2}{*}{ Gender } & Male & 14 & $58.3 \%$ & 10 & $41.7 \%$ & 24 & \multirow{2}{*}{0.49} \\
\hline & Female & 13 & $68.4 \%$ & 6 & $31.6 \%$ & 19 & \\
\hline
\end{tabular}

Table 5: Differences in characteristics between positive and negative microbiological blood cultures

\section{Discussion}

To our knowledge, this is the first prospective study that compares the diagnostic accuracy of PCT, ESR and CRP as individual and combined predictors of bacterial sepsis. We found that PCT level, on its own, was significantly the best early biomarker in predicting bloodstream bacterial infections. On the other hand, relying on negative PCT and ESR, in combination, was the best way to exclude a bacterial cause of sepsis. 


\begin{tabular}{|c|c|c|c|c|c|c|c|c|}
\hline Test(s) & $\begin{array}{l}\text { Sensitivity } \\
\text { (CI) }\end{array}$ & $\begin{array}{l}\text { Specificity } \\
\text { (CI) }\end{array}$ & $\operatorname{PLR}(\mathrm{CI})$ & $\operatorname{NLR}(\mathrm{CI})$ & OR (CI) & PPV (CI) & NPV (CI) & $P$ value \\
\hline PCT & $\begin{array}{l}74.1 \%(0.55- \\
0.87)\end{array}$ & $\begin{array}{c}68.8 \%(0.44- \\
0.86)\end{array}$ & $\begin{array}{c}2.37(1.11- \\
5.07)\end{array}$ & $\begin{array}{c}0.377(0.18- \\
0.77)\end{array}$ & $\begin{array}{c}6.286(1.61- \\
24.57)\end{array}$ & $\begin{array}{c}80 \%(59.3- \\
93.17)\end{array}$ & $\begin{array}{c}61.11 \%(35.75- \\
82.7)\end{array}$ & 0.006 \\
\hline CRP & $\begin{array}{c}66.7 \%(0.48- \\
0.81)\end{array}$ & $\begin{array}{c}37.5 \%(0.18- \\
0.61)\end{array}$ & $\begin{array}{c}1.067(0.67- \\
1.7)\end{array}$ & $\begin{array}{l}0.889(0.39- \\
2.03\end{array}$ & $1.2(0.33-4.36)$ & $\begin{array}{c}64.29 \%(44.07- \\
81.36)\end{array}$ & $\begin{array}{c}40 \%(16.34- \\
67.71)\end{array}$ & 0.78 \\
\hline ESR & $\begin{array}{c}70.4 \%(0.52- \\
0.84)\end{array}$ & $\begin{array}{c}37.5 \%(0.18- \\
0.61)\end{array}$ & $\begin{array}{c}1.126(0.72- \\
1.77)\end{array}$ & $\begin{array}{c}0.790(0.34- \\
1.87)\end{array}$ & $\begin{array}{c}1.425(0.39- \\
5.26)\end{array}$ & $\begin{array}{c}65.52 \%(45.67- \\
82.06)\end{array}$ & $\begin{array}{c}42.86 \%(17.66- \\
71.14)\end{array}$ & 0.59 \\
\hline PCT \& CRP & $\begin{array}{c}51.9 \%(0.34- \\
0.69)\end{array}$ & $\begin{array}{c}81.3 \%(0.57- \\
0.93)\end{array}$ & $\begin{array}{l}2.765(0.94- \\
8.17)\end{array}$ & $\begin{array}{c}0.593(0.37- \\
0.94)\end{array}$ & $\begin{array}{c}4.667(1.08- \\
20.19)\end{array}$ & $\begin{array}{c}82.35 \%(56.57- \\
96.2)\end{array}$ & $\begin{array}{c}50 \%(29.93- \\
70.07)\end{array}$ & 0.032 \\
\hline PCT \& ESR & $\begin{array}{c}59.3 \%(0.41- \\
0.75)\end{array}$ & $\begin{array}{c}81.3 \%(0.57- \\
0.93)\end{array}$ & $\begin{array}{c}3.160(1.09- \\
9.18)\end{array}$ & $\begin{array}{c}0.501(0.3- \\
0.84)\end{array}$ & $\begin{array}{c}6.303(1.45- \\
27.45)\end{array}$ & $\begin{array}{c}82.35 \%(56.57- \\
96.2)\end{array}$ & $\begin{array}{c}50 \%(29.93- \\
70.07)\end{array}$ & 0.01 \\
\hline $\begin{array}{c}\text { PCT, CRP \& } \\
\text { ESR }\end{array}$ & $\begin{array}{c}37 \%(0.21- \\
0.56)\end{array}$ & $\begin{array}{c}81.3 \%(0.57- \\
0.93)\end{array}$ & $\begin{array}{c}1.98(0.64- \\
6.13)\end{array}$ & $\begin{array}{c}0.77(0.53- \\
1.12)\end{array}$ & $\begin{array}{c}2.55(0.58- \\
11.18)\end{array}$ & $\begin{array}{c}76.92 \%(46.19- \\
94.96)\end{array}$ & $\begin{array}{c}43.33 \% 25.46- \\
62.57)\end{array}$ & 0.2 \\
\hline
\end{tabular}

CRP: C-reactive protein, ESR: erythrocyte sedimentation rate, NLR: negative likelihood ratio, NPV: negative predictive value, OR: diagnostic odds ratio, PCT: procalcitonin, PLR: positive likelihood ratio, PPV: positive predictive value

Table 6: Diagnostic value for positive microbiological blood cultures of PCT, CRP and ESR, individually and in combinations

\begin{tabular}{|c|c|c|c|c|c|}
\hline \multirow{2}{*}{ Test Result Variable(s) } & \multirow{2}{*}{ Area } & \multirow{2}{*}{$\begin{array}{l}\text { Standard } \\
\text { Error }^{\mathrm{a}}\end{array}$} & \multirow{2}{*}{$\begin{array}{l}\text { Asymptotic } \\
\text { Significance }^{\mathrm{b}}\end{array}$} & \multicolumn{2}{|c|}{$\begin{array}{c}\text { Asymptotic 95\% Confidence } \\
\text { Interval }\end{array}$} \\
\hline & & & & Lower Bound & Upper Bound \\
\hline PCT & 0.714 & 0.084 & 0.020 & 0.550 & 0.878 \\
\hline ESR & 0.539 & 0.092 & 0.669 & 0.358 & 0.721 \\
\hline CRP & 0.521 & 0.092 & 0.821 & 0.340 & 0.702 \\
\hline PCT and CPR positive & 0.666 & 0.085 & 0.072 & 0.500 & 0.831 \\
\hline PCT and ESR positive & 0.703 & 0.082 & 0.028 & 0.542 & 0.863 \\
\hline PCT, CPR and ESR positive & 0.591 & 0.089 & 0.321 & 0.418 & 0.765 \\
\hline
\end{tabular}

a: under the nonparametric assumption; b: Null hypothesis: true area $=0.5$

Table 7: Area under the curves (AUC) for positive microbiological blood cultures of PCT, CRP and ESR, individually and in combinations

A dilemma exists in the early differentiation between an infectious from a non-infectious cause of sepsis. Such dilemma is present due to the lack of a reliable, ready available and quick biomarker of bacterial sepsis. Therefore, a posed predicament is whether to empirically start antibiotics early in septic patients or to defer starting antibiotics until the results of microbiological blood cultures. Biomarkers such as CRP and ESR have been used in attempting to make such decision. The drawbacks of such biomarkers are their lack of reliability in diagnosing bacterial bloodstream infections [13-16]. Consequently, recent studies have proposed the use of PCT as an alternative new biomarker $[17,18]$.

The role of PCT as a valuable marker of SIRS and sepsis has already been established. In the early assessment of bacteremia, PCT levels should be interpreted with correlation to the patients' clinical status and other laboratory tests. Procalcitonin levels were shown to be significantly higher in patients with positive blood cultures, and were a better predictor of bacterial sepsis than CRP and WBC [17]. Arai et al. 2014 [19] investigated the relational contribution of PCT, WBC, CRP \& platelets in predicting blood culture results of 422 specimens, and found that blood culture results were affected by PCT and platelet levels, but not WBC and CRP [19].

Procalcitonin has also been compared with other biomarkers in predicting positive blood cultures. Naffaa et al. 2014 [20] found that PCT is significantly better than interleukin 6 in predicting positive blood cultures [20].

Although a cut-off value of PCT in diagnosing sepsis has not been determined, recent research demonstrated a higher likelihood of sepsis in levels above $0.5 \mathrm{ng} / \mathrm{mL}$. A prospective study of 105 critically ill patients recommended the inclusion of a cut-off level for PCT in the diagnostic criteria and definitions of sepsis [21]. Nevertheless, PCT has been correlated with eGFR, therefore, a decline in renal function warrants a careful interpretation of PCT level. Hattori et al 2014 [17] proposed a higher PCT cut-off value in patients with a decreased eGFR [17].

When considering the reported sensitivity and specificity of PCT, CRP and ESR, a large discrepancy exists. Studies of PCT reported sensitivity and specificity ranges of 74.8-100 and 70-100\%, respectively [2]. On the other hand, whilst the wide use of CRP and ESR as adjunctive serum biomarkers of inflammation and sepsis, many studies have questioned their roles, particularly due to an inconsistency in their reported diagnostic accuracy parameters [13-15].

In comparing PCT, CRP and ESR, individually and in combinations, we found that PCT was the best predictor of a positive blood culture with a sensitivity of $74.1 \%$ and PPV of $80 \%$ (Table 6). Moreover, although PCT, in combination with CRP, ESR or both, showed the highest specificity; $81.3 \%$, low PCT levels remain superior in excluding bacterial sepsis; specificity of $68.8 \%$ and NPV of $61.11 \%$ (Table 6). Therefore, even though early assessment of bacteremia mandates correlation of PCT with other laboratory markers such as CRP and ESR, PCT remains the best biomarker in diagnosing or excluding bacterial sepsis. 
A strong limitation to our study is our small sample size. This is attributed to the scarce availability of PCT-Q assays in our centre. Therefore, we stopped enrolling patients once statistical significance was achieved. To validate our results, further large-scale prospective studies could be done in the future. Another limitation in our study was the use of a semi-quantitative method of detecting PCT levels. At the time of study, a quantitative method of detecting PCT levels was unavailable. Optimal cut-off levels of PCT in the diagnosis of sepsis should be investigated, with special considerations to various clinical conditions and co-morbidities, such as renal failure. This can be done using a quantitative method of detecting PCT rather than the semi-quantitative method used in our study. Finally, future studies should focus on implementing the use of PCT in the diagnostic criteria of sepsis.

\section{Conclusion}

In conclusion, careful evaluation of the patients' clinical status, PCT and other laboratory markers are crucial in the early assessment of bacteremia. Although PCT levels are the best predictors of bacterial sepsis, interpretation should be done carefully when deciding the empiric use of antibiotics.

\section{Acknowledgement}

We would like to thank Maj. Gen. Prof. Khalid Bin Ali Al-Khalifa for his continuous support and encouragement towards research at the Royal Medical Services, Bahrain Defense Force hospital. We thank Dr. Marwa AlBaharna for her inputs and interest in our research project. Last, but not least, we thank Ms. Majida Basheer for the assistance she provided at the laboratory.

\section{References}

1. Vincent JL, Sakr Y, Sprung CL, Ranieri VM, Reinhart K, et al. (2006) Sepsis in European intensive care units: results of the SOAP study. Crit Care Med 34: $344-53$.

2. Chan T, Gu F (2011) Early diagnosis of sepsis using serum biomarkers. Expert Rev Mol Diagn 11: 487-96.

3. Tang BM, Eslick GD, Craig JC, McLean AS (2007) Accuracy of procalcitonin for sepsis diagnosis in critically ill patients: systematic review and meta-analysis. Lancet Infect Dis 7: 210-7.

4. Brunkhorst FM, Wegscheider K, Forycki ZF, Brunkhorst R (2000) Procalcitonin for early diagnosis and differentiation of SIRS, sepsis, severe sepsis, and septic shock. Intensive Care Med 26: S148-52.

5. Levy MM, Fink MP, Marshall JC, Abraham E, Angus D, et al. (2003) 2001 SCCM/ESICM/ACCP/ATS/SIS International Sepsis Definitions Conference. Crit Care Med 31: 1250-6.

6. Al-Nawas B, Krammer I, Shah PM (1996) Procalcitonin in diagnosis of severe infections. Eur J Med Res 1: 331-3.

7. Meisner M, Brunkhorst FM, Reith HB, Schmidt J, Lestin HG, et al. (2000) Clinical experiences with a new semi-quantitative solid phase immunoassay for rapid measurement of procalcitonin. Clin Chem Lab Med 38: 989-95.

8. Aikawa N, Fujishima S, Endo S, Sekine I, Kogawa K, et al. (2005) Multicenter prospective study of procalcitonin as an indicator of sepsis. J Infect Chemother 11: 152-9.

9. Meisner M, Adina H, Schmidt J (2006) Correlation of procalcitonin and C-reactive protein to inflammation, complications, and outcome during the intensive care unit course of multiple-trauma patients. Crit Care 10: R1.

10. Garnacho-Montero J, Huici-Moreno MJ, Gutiérrez-Pizarraya A, López I, Márquez-Vácaro JA, et al. (2014) Prognostic and diagnostic value of eosinopenia, Creactive protein, procalcitonin, and circulating cell-free DNA in critically ill patients admitted with suspicion of sepsis. Crit Care 18 : R116.

11. Maruna P, Nedelníková K, Gürlich R (2000) Physiology and genetics of procalcitonin. Physiol Res 49: S57-61.

12. Dandona P, Nix D, Wilson MF, Aljada A, Love J, et al. (1994) Procalcitonin increase after endotoxin injection in normal subjects. J Clin Endocrinol Metab 79: 1605-8.

13. Nelson GE, Mave V, Gupta A (2014) Biomarkers for sepsis: a review with special attention to India. Biomed Res Int 2014: 10.1155/2014/264351.

14. Ho KM, Lipman (2009) An update on C-reactive protein for intensivists. Anaesth Intensive Care 37: 234-41.

15. Kim KE, Han JY (2010) Evaluation of the clinical performance of an automated procalcitonin assay for the quantitative detection of bloodstream infection. Korean J Lab Med 30: 153-9.

16. Bates DW, Sands K, Miller E, Lanken PN, Hibberd PL, et al. (1997) Predicting bacteremia in patients with sepsis syndrome. Academic Medical Center Consortium Sepsis Project Working Group. J Infect Dis 176: 1538-51.

17. Hattori T, Nishiyama H, Kato H, Ikegami S, Nagayama M, et al. (2014) Clinical value of procalcitonin for patients with suspected bloodstream infection. Am J Clin Pathol 141: 43-51.

18. Albrich WC, Mueller B (2011) Predicting bacteremia by procalcitonin levels in patients evaluated for sepsis in the emergency department. Expert Rev Anti Infect Ther 9: 653-6.

19. Arai T, Kumasaka K, Nagata K, Okita T, Oomura T, et al. (2014) Prediction of blood culture results by measuring procalcitonin levels and other inflammatory biomarkers. Am J Emerg Med 32: 330-3.

20. Naffaa M, Makhoul BF, Tobia A, Kaplan M, Aronson D et al. (2014) Procalcitonin and interleukin 6 for predicting blood culture positivity in sepsis. Am J Emerg Med 32: 448-51.

21. Giamarellos-Bourboulis EJ, Giannopoulou P, Grecka P, Voros D, Mandragos K, et al. (2004) Should procalcitonin be introduced in the diagnostic criteria for the systemic inflammatory response syndrome and sepsis? J Crit Care 19: 152-7. 


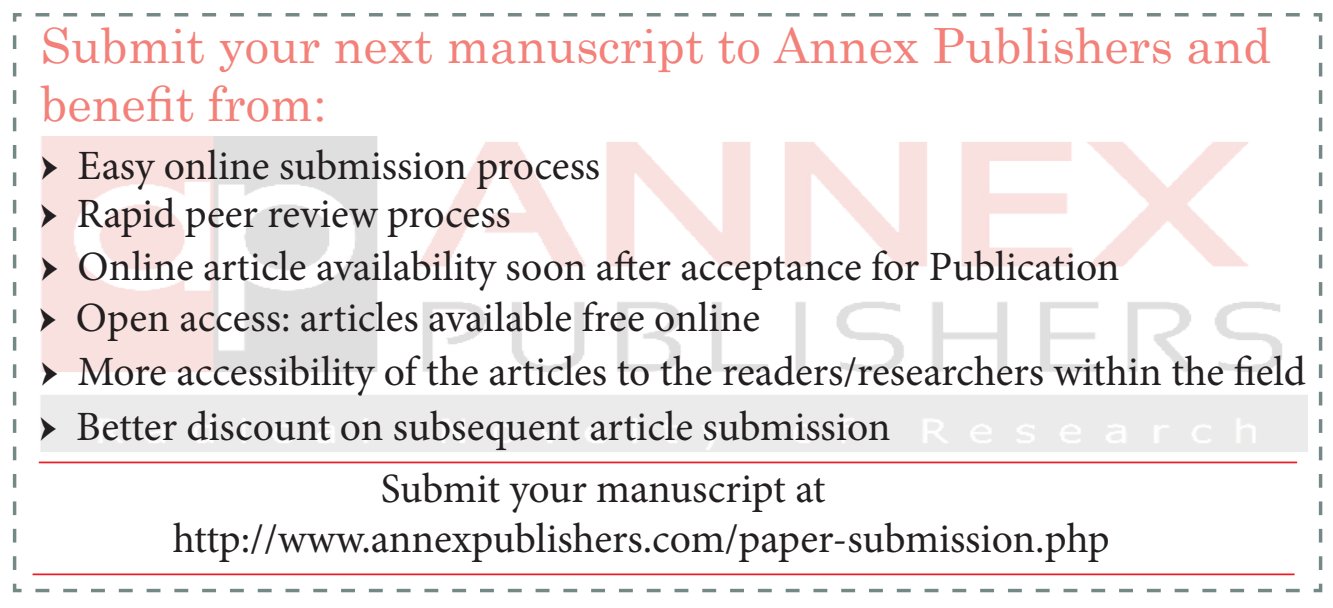

